\title{
Dependencia de la velocidad de reacción de la anhidrasa carbónica en los eritrocitos
}

\author{
Dependence of the reaction rate of carbonic anhydrase in erythrocytes \\ Linda J. Aguilar-López ${ }^{a}$, Martha G. Martínez-Ángeles ${ }^{b}$, María C. Pérez-Lugo ${ }^{c}$
}

\begin{abstract}
:
Carbonic anhydrase is an enzyme that belongs to a family of metalloenzymes, enzymes that contain one or more metal atoms as a functional component of the enzyme and that catalyze the rapid conversion of carbon dioxide and water to bicarbonate and hydrogen ions, a reaction that occurs more slow in the absence of the catalyst. It is a zinc dependent metalloenzyme, it plays an important role in the reversible catalysis of the hydration of $\mathrm{CO}_{2}$ to form $\mathrm{HCO}_{3}{ }^{-}$and $\mathrm{H}^{+}$. Of the five families described, AC-a is found in humans, it is divided into four subgroups and fourteen isoforms. Its wide cell distribution gives it versatile functionality. ${ }^{1}$ Recently reported studies lead us to question the role that this enzyme plays in the genesis, control and progress of lung diseases, such as asthma, COPD (Chronic Obstructive Pulmonary Disease) and even atherosclerosis and cancer.
\end{abstract}

\section{Keywords:}

Hemoglobin, carbonic anhydrase, catalyst.

\section{Resumen:}

La anhidrasa carbónica es una enzima que pertenece a una familia de metaloenzimas, enzimas que contienen uno o más átomos metálicos como componente funcional de la enzima y que catalizan la conversión rápida de dióxido de carbono y agua a bicarbonato y iones hidrògeno, una reacción que ocurre más lenta en ausencia del catalizador. Es una metaloenzima zinc dependiente, juega un papel importante en la catálisis reversible de la hidratación del $\mathrm{CO}_{2}$ para formar $\mathrm{HCO}_{3}{ }^{-} \mathrm{y} \mathrm{H}^{+}$. De las cinco familias descritas, la $\mathrm{AC}^{-} \mathrm{a}$ se encuentra en los seres humanos, ésta se divide en cuatro subgrupos y catorce isoformas. Su amplia distribución celular, le confiere una versátil funcionalidad. Los estudios reportados recientemente nos llevan a cuestionarnos acerca del papel que esta enzima juega en la génesis, el control y progreso de las enfermedades pulmonares, como asma, EPOC (Enfermedad Pulmonar Obstructiva Crònica) e inclusive la aterosclerosis y càncer.

\section{Palabras Clave:}

Hemoglobina, anhidrasa carbónica, catalizador.

\footnotetext{
a Universidad Autónoma del Estado de Hidalgo, https://orcid.org/0000-0002-0019-9078, Email: ag391218@uaeh.edu.mx

b Autor de correspondencia, Universidad Autónoma del Estado de Hidalgo, https://orcid.org/0000-0002-3914-9350, Email: ma434269@uaeh.edu.mx

c Universidad Autónoma del Estado de Hidalgo, https://orcid.org/0000-0003-4161-2831, Email: pe353627@uaeh.edu.mx
} 


\section{Introducción}

Anselme Payen, químico, es conocido por el descubrimiento de la primera enzima en 1833, y por sus trabajos sobre la celulosa y el papel. ${ }^{6}$

Las enzimas son proteínas que tienen la capacidad de catalizar una reacción. Estas pueden procesar muchas reacciones a causa de los grupos $\mathrm{R}$ en los aminoácidos. La anhidrasa carbónica (AC) es una metaloenzima dependiente de zinc, ya que es esencial para su actividad catalítica. La AC cataliza la reacción reversible del dióxido de carbono $\left(\mathrm{CO}_{2}\right)$ a ion bicarbonato $\left(\mathrm{HCO}_{3}{ }^{-}\right)$y viceversa:

$\mathrm{CO}_{2}+\mathrm{H}_{2} \mathrm{O} \rightarrow \mathrm{H}_{2} \mathrm{CO}_{3} \rightarrow \mathrm{HCO}_{3}^{-}+\left(\mathrm{H}^{+}\right) \mathrm{O}\left(\mathrm{HCO}_{3}{ }^{-}\right)+\left(\mathrm{H}^{+}\right) \rightarrow$ $\mathrm{CO}_{2}+\mathrm{H}_{2} \mathrm{O} \rightarrow \mathrm{H}_{2} \mathrm{CO}_{3}$

Esta enzima se encuentra en los eritrocitos humanos junto con la hemoglobina. La cinética enzimática estudia la velocidad de las reacciones catalizadas por enzimas. La velocidad puede determinarse midiendo la aparición de los productos o la desaparición de los reactivos. La velocidad de reacción de una enzima se puede analizar por medio de la ecuación de Michaelis-Menten.

\section{Dependencia la velocidad de reacción de la anhidrasa carbónica en los eritrocitos}

\subsection{Enzimas}

Anselme Payen fue el químico que descubrió la primera enzima en $1833 .^{6}$

Las enzimas son proteínas que tienen la capacidad de catalizar una reacción, no obstante, algunos ARN tienen actividad catalítica, por ejemplo: ribozimas.

Las enzimas pueden procesar muchas reacciones a causa de los grupos $\mathrm{R}$ en los aminoácidos, también generan un ambiente idóneo para que estas reacciones ocurran. Se lleva a cabo mediante la creación en su interior de un sitio activo (ambiente idóneo), donde la enzima realiza su función catalítica y es el lugar de unión con el sustrato. Generar un ambiente idóneo hace referencia a la orientación adecuada de los sustratos para que realicen su función y la formación transitoria inestable que permite la transición adecuada de sustrato a producto.

Es decir, para que una molécula A reaccione y pase a ser $B$ es necesario que alcance un estado energético específico y de esa forma alcance ese estado de transición, ahí radica la importancia de los catalizadores, ya que, si no hay, la demanda de energía es mayor y mientras más energía se requiera, la reacción tardará más tiempo de lo habitual. ${ }^{2}$

La presencia del catalizador provoca una disminución en la energía de activación requerida, de manera que la velocidad de reacción con la que se desarrolla la misma.
Sin embargo, los catalizadores no aportan energía a la reacción, sino que desvían la energía para cubrir la demanda energética y éstos no se pierden en la reacción.
Reacción no catalizada

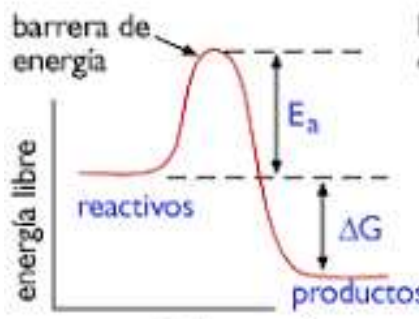

curso de la reacción
Reacción catalizada

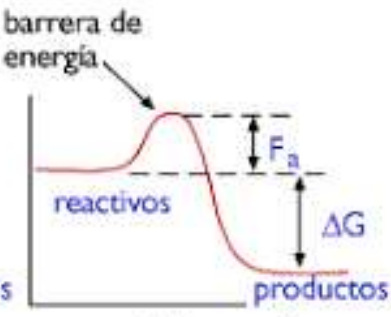

curso de la reacción
Figura 1. Presencia del catalizador en una reacción química. $^{4}$

\subsection{Actividad enzimática}

Las reacciones, sean catalizadas o no, dependen de las leyes termodinámicas, estas leyes permiten deducir si una reacción se desarrolla o no, de forma espontánea es el cambio en la energía libre de Gibbs, $(\Delta G)$ deducido de la segunda ley de la termodinámica (una reacción es espontánea si la entropía global del universo aumenta), que mide la capacidad de un sistema para desarrollar un trabajo. Para que se realice la transformación de una molécula se le denomina sustrato (S), el resultado se denomina producto $(P)$, si el cambio de energía libre de Gibbs es negativo, implica que la energía libre del producto sea menor que la del sustrato.

En una reacción química la conversión de sustrato en producto requiere una situación energética intermedia que se denomina "estado de transición", donde el nivel de energía es superior al del sustrato o del producto.

La diferencia entre el nivel de energía basal y la correspondiente al estado de transición se denomina "energía de activación" y cuanto más alta sea, menor será la velocidad de reacción. Es la determinación de la velocidad de reacción de una enzima y cómo cambia a condiciones diferentes. ${ }^{12}$

Algunas de las condiciones que afectan a la velocidad de reacción son: la temperatura, $\mathrm{pH}$, concentración de sustrato (esta es la que más afecta la velocidad de reacción) y por último tenemos la presencia de inhibidores.

Algunos ejemplos de los tipos de enzimas son: 
Tabla 1. Clasificación de enzimas. ${ }^{14}$

\begin{tabular}{|l|l|l|}
\hline Clase & Tipo de reacción & Ejemplo \\
\hline $\begin{array}{l}\text { Oxidorreductasas } \\
\text { (22 Subclases) }\end{array}$ & Oxido-reducción & Lactato Deshidrogenasa \\
\hline $\begin{array}{l}\text { Transferasas } \\
\text { (9 Subclases) }\end{array}$ & Transferencia de grupos & $\begin{array}{l}\text { Glutamato Oxalacetato } \\
\text { Transaminasa }\end{array}$ \\
\hline $\begin{array}{l}\text { Hidrolasas } \\
\text { (13 Subclases) }\end{array}$ & Hidrólisis (agua) & Quimotripsina \\
\hline $\begin{array}{l}\text { Liasas } \\
\text { (7 Subclases) }\end{array}$ & $\begin{array}{l}\text { Adición o eliminación de grupos } \\
\text { para formar dobles enlaces }\end{array}$ & Fumarasa \\
\hline $\begin{array}{l}\text { Isomerasas } \\
\text { (6 Subclases) }\end{array}$ & $\begin{array}{l}\text { Isomerización (transferencia de } \\
\text { grupos intramolecular }\end{array}$ & Triosa fosfato isomerasa \\
\hline $\begin{array}{l}\text { Ligasas } \\
\text { (6 Subclases) }\end{array}$ & $\begin{array}{l}\text { Ligación de dos sustratos } \\
\text { implicando hidrólisis de ATP }\end{array}$ & Piruvato carboxilasa \\
\hline
\end{tabular}

\subsection{Cinética enzimática}

La cinética enzimática estudia la velocidad de las reacciones catalizadas por enzimas. La velocidad puede determinarse midiendo la aparición de los productos o la desaparición de los reactivos. ${ }^{7}$

Forma en que se analiza el cambio de velocidad de una enzima:

- Medición de la velocidad inicial de una enzima en tiempos cortos (menos de 60s).

- Utilización de una concentración de sustrato elevada respecto a la cantidad de enzima que hay (generalmente por una molécula de enzima se utiliza entre 100,000 y $1,000,000$ de moléculas de sustrato).

La velocidad de reacción de una enzima se puede analizar por medio de la ecuación de Michaelis-Menten, quienes explicaron el comportamiento de las enzimas en 1912 por medio de su fórmula:

Ec. 1

$$
\mathrm{E}+\mathrm{S} \leftrightarrow \mathrm{ES} \leftrightarrow \mathrm{EP} \leftrightarrow \mathrm{E}+\mathrm{P}
$$
E: Enzima
S: Sustrato
ES: Complejo enzima-sustrato
EP: Complejo enzima-producto
$P$ : Producto

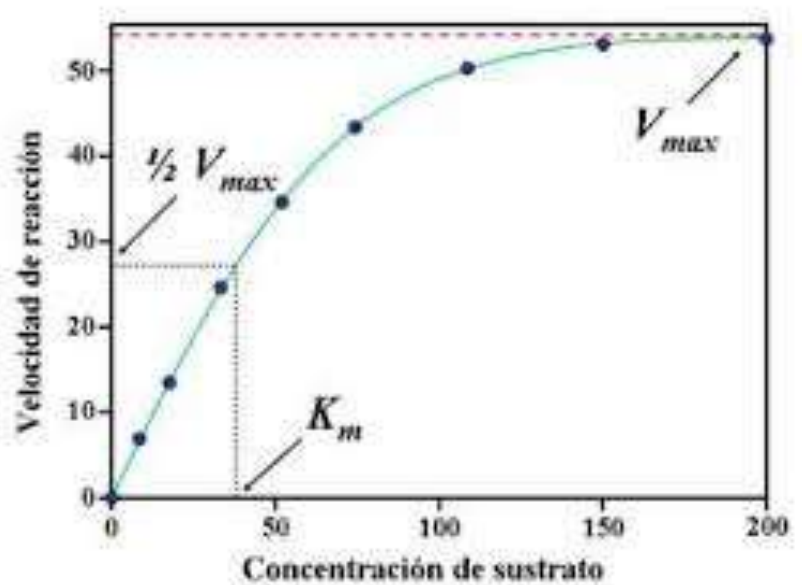

Figura 2: Comportamiento enzimático según MichaelisMenten. ${ }^{16}$

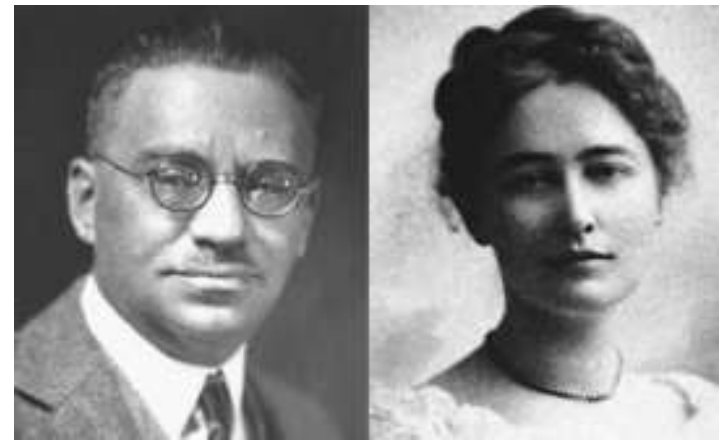

Figura 3: Michaelis-Menten ${ }^{11}$

Esta trata de explicar que al inicio del proceso de adición del sustrato la enzima tendrá un cambio muy notorio, ya que estará libre para unirse al sustrato, conforme se continúan uniendo se formará el complejo ES, después pasará al complejo EP, donde ya habrá una mayor cantidad de producto, causando un acercamiento drástico a Vmax, que es la velocidad máxima que alcanza una enzima aproximadamente. Posteriormente se van a separar.

La forma matemática que se utilizó para demostrar este comportamiento en 1913 fue la ecuación de MichaelisMenten, la cual permite conocer y entender por qué algunas enzimas llegan a un punto en el que la Vmax aparentemente ya no avanza. ${ }^{9}$

Ecuación de Michaelis-Menten:

Ec. $2 \quad V o=\frac{\operatorname{Vmax}(S)}{K m+(S)}$

Vo: Velocidad inicial

Vmax: Velocidad máxima

$\mathrm{Km}$ : Constante de Michaelis-Menten

(S): Concentración de sustrato

\subsection{La velocidad de reacción, estructura y función de la anhidrasa carbónica en los eritrocitos.}

Es una metaloenzima zinc dependiente, esencial para su actividad catalítica. La AC cataliza la reacción reversible del dióxido de carbono $\left(\mathrm{CO}_{2}\right)$ a ion bicarbonato $\left(\mathrm{HCO}_{3}{ }^{-}\right)$y viceversa:

$$
\begin{aligned}
& \mathrm{CO}_{2}+\mathrm{H}_{2} \mathrm{O} \rightarrow \mathrm{H}_{2} \mathrm{CO}_{3} \rightarrow \mathrm{HCO}_{3}^{-}+\left(\mathrm{H}^{+}\right) \mathrm{o}\left(\mathrm{HCO}_{3}^{-}\right)+\left(\mathrm{H}^{+}\right) \rightarrow \\
& \mathrm{CO}_{2}+\mathrm{H}_{2} \mathrm{O} \rightarrow \mathrm{H}_{2} \mathrm{CO}_{3}
\end{aligned}
$$




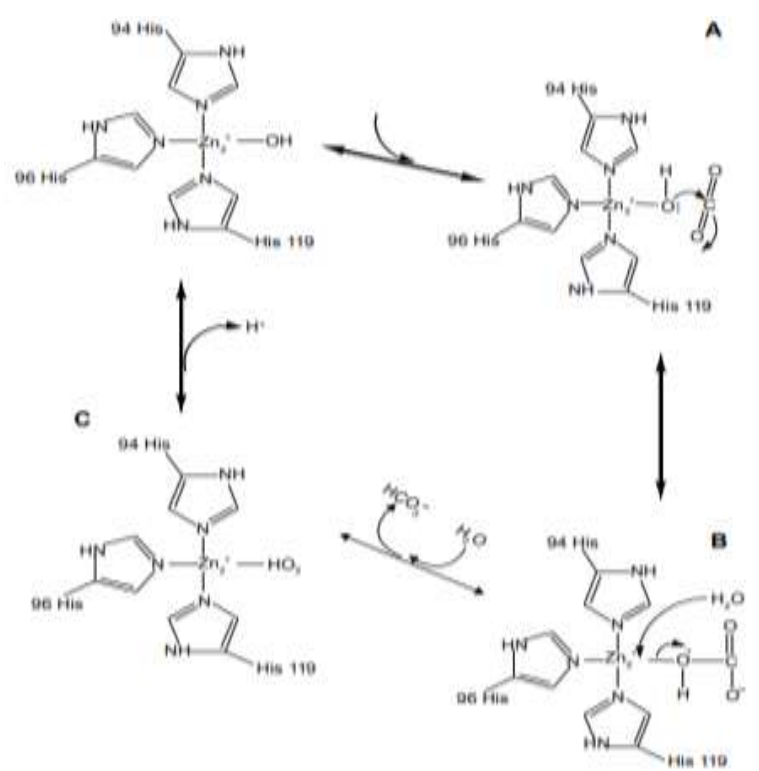

Figura 4: Anhidrasa carbónica y la importancia del zinc que la compone. ${ }^{13}$

Su amplia distribución celular, le confiere una versátil funcionalidad.

Los estudios reportados recientemente nos llevan a cuestionarnos acerca del papel que esta enzima juega en la génesis, el control y progreso de las enfermedades pulmonares, como asma, EPOC e inclusive la aterosclerosis y cáncer.

En este caso vamos a estudiar la anhidrasa carbónica, cómo actúa en el eritrocito y de qué depende.

Las isoformas I y II se encuentran en el eritrocito (célula sanguínea que posee hemoglobina en su interior y se encarga de la distribución de oxígeno en el organismo). La isoforma I está presente en un $85 \%$ y en concentraciones sanguíneas de $150 \mathrm{mM}$; mientras que la

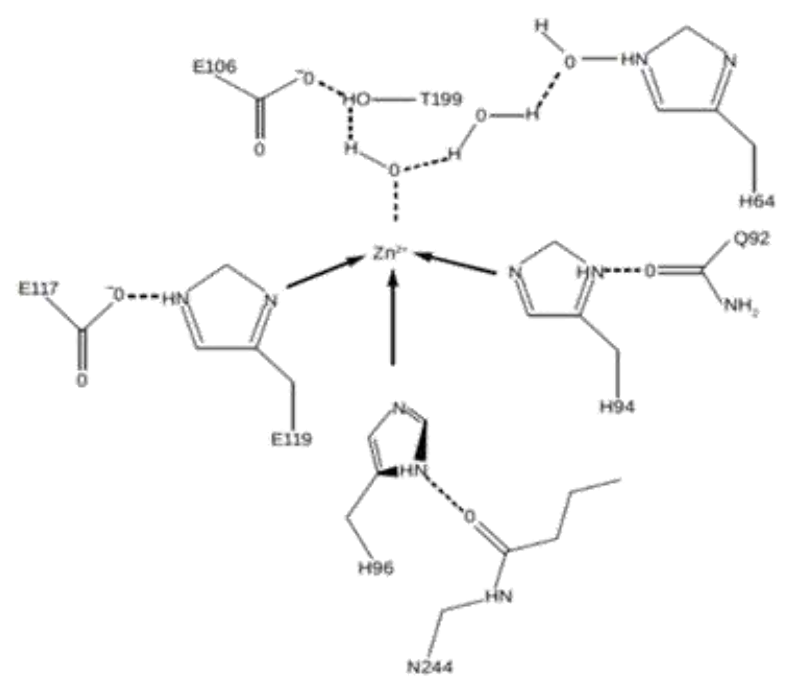

Figura 5: Estructura de la anhidrasa carbónica. Se observa la molécula de zinc en el sitio activo, ésta coordina con los anillos imidazol de histidina 94,96 y $119 .^{10}$ isoforma II se encuentra en menor proporción, tiene una mayor actividad y su tasa de recambio es de 106s-1 a $25^{\circ} \mathrm{C}$ y a un $\mathrm{pH}$ de $9.5 .{ }^{15}$

Las concentraciones fisiológicas de cloro $(80 \mathrm{mM})$. Esta enzima contribuye a la excreción del $\mathrm{CO}_{2}$ mediante su hidratación y con secuente conversión a $\mathrm{HCO}_{3}$, durante la hidratación del $\mathrm{CO}_{2}$ se producen también protones que son amortiguados por la hemoglobina $(\mathrm{Hb})$, mientras que los iones $\mathrm{HCO}_{3}$ son transportados hacia el plasma vía la proteína de la banda $3 .{ }^{10}$

Esta reacción se revierte a nivel alveolar, donde el $\mathrm{HCO}_{3}$ se deshidrata en el eritrocito produciendo $\mathrm{CO}_{2}$ que es capaz de difundirse a través de un gradiente de presión por la membrana alveolocapilar. Unido a dicho compuesto, el eritrocito favorece el abastecimiento de oxígeno $\left(\mathrm{O}_{2}\right)$ durante su paso a través del lecho capilar mediante el efecto Bohr.

Actualmente, se sabe que es una de las células más especializadas del organismo, en la que más del $95 \%$ de las proteínas citoplasmáticas corresponde a la $\mathrm{Hb}$ y cuya fuente principal de energía es proporcionada por glucólisis (ruptura de la molécula de glucosa que da como producto dos moléculas de piruvato), la cual mantiene el glutatión en su forma reducida protegiendo de esta manera a su membrana, así como a los grupos sulfhídrilo de la $\mathrm{Hb}$, del daño generado por las especies reactivas del oxígeno y nitrógeno. ${ }^{8}$

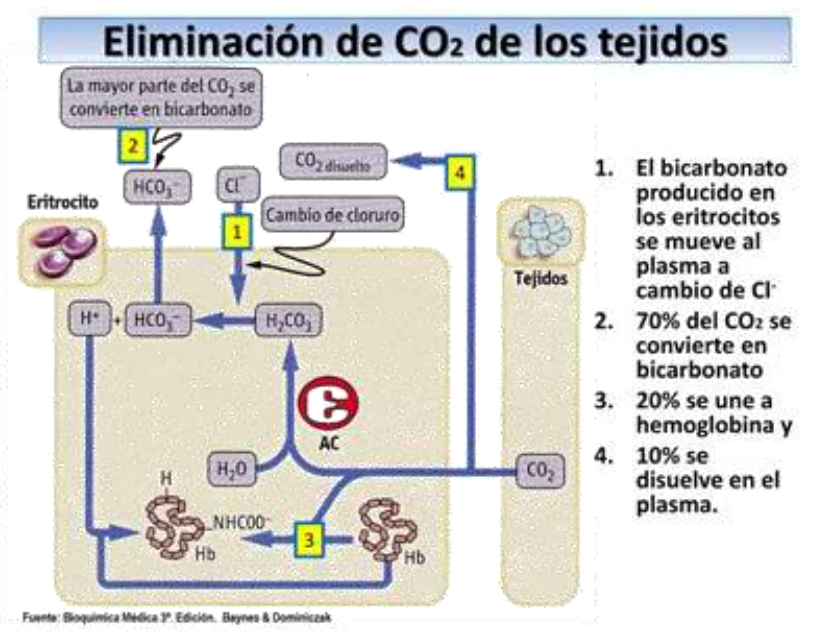

Figura 6: El comportamiento de la anhidrasa carbónica durante la eliminación de $\mathrm{CO}_{2}$ desde los tejidos. ${ }^{3}$

Es decir, que cuando el $\mathrm{CO}_{2}$ que se halla en los tejidos se va a eliminar, este pasará al plasma y el eritrocito lo va a capturar por medio de la transformación en el que la anhidrasa carbónica lo va a transformar en ácido carbónico. El $20 \%$ de este se unirá a la hemoglobina (proteína de estructura cuaternaria que es afina al $\mathrm{O}_{2}$ ) como barmino (cuando ya se ha unido con la hemoglobina el $\mathrm{CO}_{2}$ ), después se va a ionizar en bicarbonato $\mathrm{H}^{+}$, posteriormente el bicarbonato se va a 
expulsar al plasma y un ion cloruro va a entrar (desplazamiento de cloruro).

Una vez sucedido esto, el eritrocito se va comenzar a acidificar, por ende, la hemoglobina (que contiene histidinas) va a capturar los $\mathrm{H}^{+}$y va a regular el $\mathrm{pH}$.

\subsection{Eliminación del (dióxido de carbono) $\mathrm{CO}_{2}$ con la espiración}

La hemoglobina liberará al catión hidrógeno $\left(\mathrm{H}^{+}\right)$ capturado, este ion $\mathrm{H}^{+}$se unirá con el bicarbonato para formar el ácido carbónico. La anhidrasa carbónica los va a transformar en $\mathrm{CO}_{2}$ y $\mathrm{H}_{2} \mathrm{O}$, de esa manera se va a liberar el $\mathrm{CO}_{2}$ en los pulmones. Cuando se liberan los iones $\mathrm{H}^{+}$, puede alcalinizarse el eritrocito, pero esto no ocurrirá porque se van a liberar en el plasma. ${ }^{9}$

\section{Excreción de $\mathrm{CO}_{2}$ en el aire expirado}

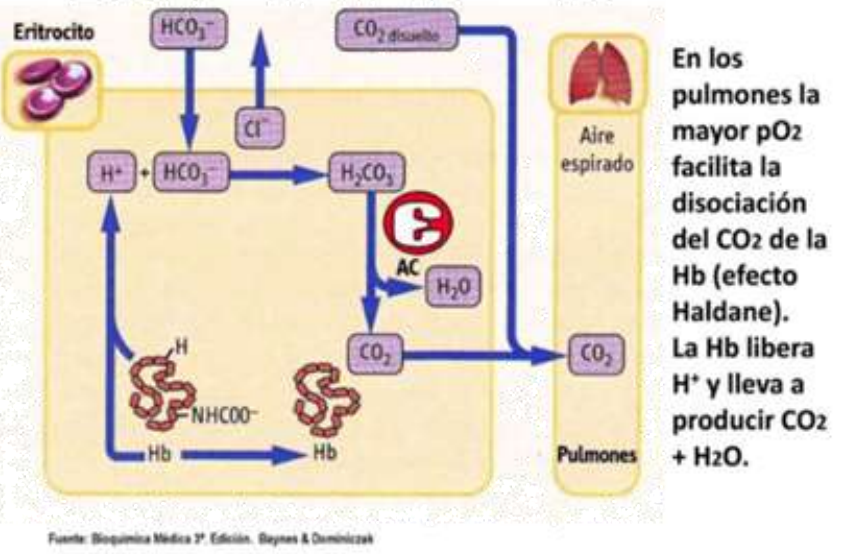

Figura 7: El comportamiento de la anhidrasa carbónica durante la eliminación de $\mathrm{CO}_{2}$ desde el aire espirado. ${ }^{3}$

\subsection{Inhibidores de Anhidrasa Carbónica (IAC)}

Se unen de una manera tetraédrica al ion de zincen su estado desprotonado por medio del átomo de nitrógeno del motivo amida de la sulfonamida, mientras que las cadenas aromáticas laterales forman puentes de hidrógeno que involucran los aminoácidos Thr199 y Glu106 del sitio activo de la enzima.

La parte aromática heterocíclica de los IAC tipo sulfonamidas interactúa con residuos hidrofílicos (residuos que poseen afinidad porel agua) e hidrofóbicos (residuos que no poseen afinidad por el agua, ejemplo, lípidos) del sitio activo, pero también puede unirse de una manera tetraédrica con el ion metal. ${ }^{1}$

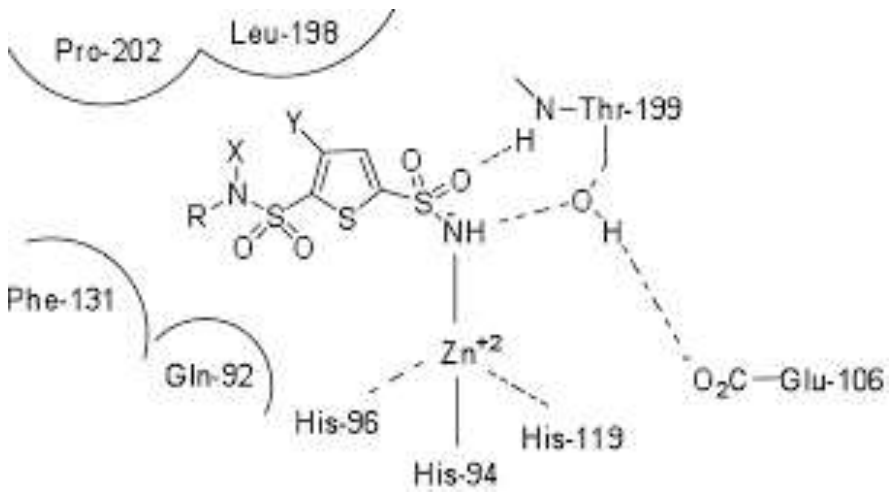

Figura 8: Sulfonamida inhibidora de anhidrasa carbónica humana, las cadenas aromáticas laterales forman puentes de hidrógeno, involucrando los aminoácidos Thr199 y Glu106 del sitio activo de la anhidrasa carbónica, interactuando con residuos hidrofílicos e hidrofóbicos del sitio activo. ${ }^{5}$ 


\section{DEPENDENCIA DE LA VELOCIDAD DE REACCIÓN DE LA ANHIDRASA CARBÓNICA EN LOS ERITROCITOS.}

\section{¿QUÉ ES UNA ENZIMA?}

Las enzimas son proteinas que tienen la capacidad de catalizar una reacción. Estas pueden procesar muchas reacciones a causa de los grupos R en los aminoácidos.

\section{¿QUÉ ES LA ANHIDRASA CARBÓNICA?}

La anhidrasa carbónica (AC) es una metaloenzima dependiente de zinc, ya que es esencial para su actividad catalitica.

La $A C$ cataliza la reacción reversible del dióxido de carbono $\left(\mathrm{CO}_{2}\right)$ a ion bicarbonato $\left(\mathrm{HCO}_{3}-\right)$ y viceversa: $\left.\mathrm{CO} 2+\mathrm{H}_{2} \mathrm{O} \rightarrow \mathrm{H} \mathrm{CCO}_{2} \rightarrow \mathrm{HCO}_{2}+\langle\mathrm{H}+\} \mathrm{O}\langle\mathrm{HCO}\rangle\right\}+\langle\mathrm{H}+\}$ $\rightarrow \mathrm{CO}_{2}+\mathrm{H}_{2} \mathrm{O} \rightarrow \mathrm{H}_{2} \mathrm{CO}_{3}$

Esta enzima se encuentra en los eritrocitos humanos junto con la hemoglobina.

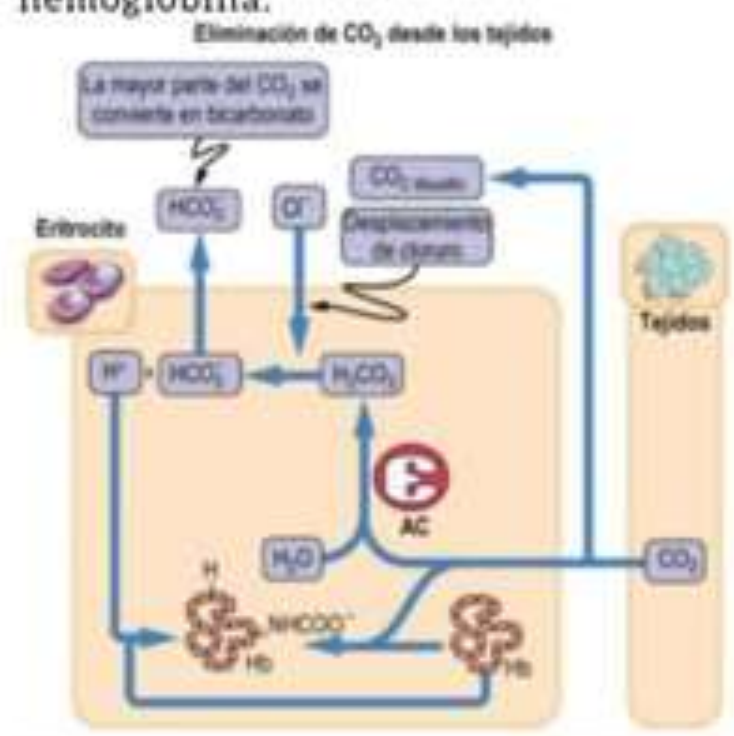

Fe 1 Ejempla de catalitación por la AC en el proceso de elminacion de $\mathrm{CO} 2$ desdet los tejidos.

\section{CINÉTICA ENZIMÁTICA}

La cinética enzimática estudia la velocidad de las reacciones catalizadas por enzimas. La velocidad puede determinarse midiendo la aparición de los productos o la desaparición de los reactivos.

La forma matemática que se utilizó para demostrar este comportamiento en 1913 fue la ecuación de Michaelis: Menten.

$$
V_{0}=\frac{V_{\max }[\mathrm{S}]}{K_{\mathrm{m}}+[\mathrm{S}]}
$$

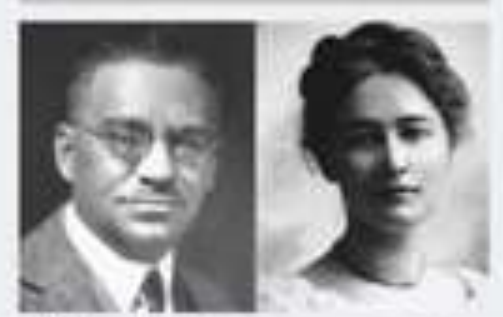

Fig? Michaeits-Menterny su ecuacon para la representación de lu cinética ensimatica

\section{REFERENCIAS BIBLIOGRAFICAS}

- Aibert L Lekninges, M. M. pood. Priapiples Ee Diopuimirs 14'EDS Letininger OMECa.

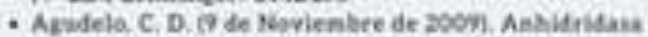
carbóntea Obtenido de Anhidtidsa cas bonica de Vixenotiein talkiparum.

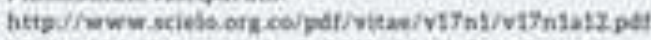

- Miclael Me Nitsan, D. L. \& CoX aotar Principios de bioquianica, 7/ED Lehninger; CMtCA.

- Mejo V, Cosmatri O. Ruainus, cunreptes basiras y ciaetica.

- En: Meis VR, Cuamatici OT, edvores, Boguimica de ins

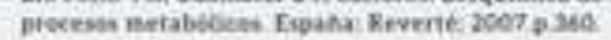





\section{Referencias}

(1)Agudelo, C. D. (2009, Noviembre 9). Anhidrasa carbònica. Retrieved from Anhidrasa carbònica de Plasmodium falciparum: http://www.scielo.org.co/pdf/vitae/v17n1/v17n1a1 2.pdf

(2)Albert L. Lehninger, M. M. (2006). Principios de Bioquìmica ( $4^{a}$ ED.) Lehninger. OMEGA.

(3)Barreno, K. K. (n.d.). Universidad de San Carlos de Guatemala. Retrieved Septiembre 25, 2020, from Control del equilibrio àcido-base: https://slideplayer.es/slide/11899461/

(4)La quìmica curiosa. (2013, Enero 29). Retrieved from Cinètica quìmica: https://quimicachemistry.files.wordpress.com/201 3/01/energc3ada_de_activacic3b3n.png

(5)López, S., \& Romero, Á. (2011). Inhibidores de la anhidrasa carbònica. Retrieved from Grupo trifluorometilo: un sustituyente importante en química medicinal: file://C:/Users/52773/Downloads/1544-2992-1SM.pdf

(6)Macho, M. (2015, Enero 06). ztfnews. Retrieved from Anselme Payen, descubridor de la primera enzima: https://ztfnews.eus/2015/01/06/anselme-payendescubridor-de-la-primeraenzima/\#: :text=El\%20qu\%C3\%ADmico\%20Ans elme \%20Payen \%20(1795,1a\%20celulosa\%20y\%2 0e1\%20papel.

(7)Mañas, J. M. (n.d.). Curso de biomolèculas. Retrieved Septiembre 29, 2020, from Cinètica enzimàtica: http://www.ehu.eus/biomoleculas/enzimas/enz3.ht $\mathrm{m}$

(8)Melo V, C. O. (2007). Enzimas: conceptos básicos y cinética. In C. O. Melo VR, Bioquímica de los procesos metabólicos. (p. p.360.). España : Reverté.
(9)Michael M. Nelson, D. L., \& COX. (2018). Principios de bioquìmica, 7/ED Lehninger. OMEGA.

(10)Monroy, L. E., \& Vargas, M. P. (2011, Marzo 11). Anhidrasa carbónica. Retrieved from Anhidrasa carbónica, nuevas perspectivas: https://www.medigraphic.com/pdfs/neumo/nt2010/nt104d.pdf

(11)Nicolàs, J. M. (2018, Noviembre 26). La verdad. Retrieved from La fascinante historiade MichaelisMenten:

https://static3.laverdad.es/www/multimedia/20181 1/24/media/cortadas/fascinante-kxhGU601670279925SzG624x385@La\%20Verdad.jpg

(12)Pérez, J. M., \& Borge, M. J. (n.d.). Actividadenzimàtica. Retrieved Septiembre 29, 2020, from Fisiologì general:

https://ocw.unican.es/pluginfile.php/879/course/se ction/967/Tema\%25202B-Bloque\%2520IEnzimas.pdf

(13)Slideshare. (n.d.). Retrieved Septiembre 29, 2020, from Anhidrasa carbònica: https://image.slidesharecdn.com/anhidrasacarbnica -nuevasperspectivas-121123112004phpapp01/95/anhidrasa-carbnica-nuevasperspectivas-3-638.jpg?cb=1353669638

(14)Universidad Complutense de Madrid . (2016, Junio 26). Clasificación enzimas. Retrieved from Tema 6. Bioquímica I, Apuntes de Bioquímica: https://static.docsity.com/documents_pages/apunte s/2016/06/26/15b1fc822b2e40d0bd1591ebcde6c8f 6.png

(15)Vargas, D. M. (2010). Departamento de Investigación en Bioquímica y Medicina Ambiental. Mèxico.

(16)Wikipedia. (n.d.). Wikipedia, la enciclopedia. Retrieved Septiembre 29, 2020, from Cinètica enzimàtica: https:/upload.wikimedia.org/wikipedia/commons/ thumb/6/67/Gr\%C3\%A1 fica_Km.png/400pxGr\%C3\%A1fica_Km.png 